\title{
NOTÍCIAS E COMENTÁRIOS
}

CORRESPONDÊNCIA. Esta seção de Arquivos de Neuro-Psiquiatria divulga opiniões e observações construtivas acerca de artigos publicados em seus últimos números. São aceitas cartas assinadas, sucintas e desprovidas de caráter pessoal, abordando aspectos objetivos dos artigos e utilizando até cinco referências bibliográficas.

Enviar sua contribuição em uma via acompanhada do respectivo disquete. Utilizar o endereço: Arquivos de $\mathrm{Neu}$ ro-Psiquiatria, Praça Amadeu Amaral 47 / 33, 01327-010 São Paulo SP.

MICHAEL PRIZE 2001/2002. Até 30 novembro 2002 acham-se abertas as inscrições para o Michael Prize 2001/ 2002, patrocinado bienalmente pela STIFTUNG MICHAEL desde 1963 e tendo como finalidade estimular investigações experimentais e clínicas sobre epilepsia. $O$ prêmio é destinado a pesquisadores com até 40 anos de idade. Os postulantes devem submeter seus manuscritos ou publicações em inglês ou em alemão, em três vias, acompanhados de três vias de seu curriculum vitae. Até quatro contribuições podem ser submetidas pelo mesmo postulante, que deve assinalar aquela que considera a principal. O prêmio se acompanha da importância de 12500 doada por NOVARTIS, conglomerado de Ciba e Sandoz. Compõem a Comissão do Prêmio os Professores: R. Canger (Milão, Itália), F. Hanefeld (Göttingen, Alemanha), E. J. Speckmann (Münster, Alemanha). Administrador Executivo: Dr. H. Reith. Informes e inscrições: Stiftung Michael, Münzkamp 5, 22339 Hamburg, Deutschland. FAX 49405381559.

CURSOS DE EXTENSÃO 2002: UNIDADE DE DEPENDÊNCIA DE DROGAS (UDED). A UDED é um setor do Departamento de Psicobiologia da Universidade Federal de São Paulo (UNIFESP). Dentre os cursos sobre dependência de drogas que a UDED programou para este ano contam ainda, em: 7 setembro cocaína / crack e outros estimulantes do sistema nervoso central; 5 outubro êxtase, maconha e outros alucihógenos; 9 novembro intervenções breves para usuários de drogas - adultos e adolescentes. Informes e inscrições: UDED / UNIFESP, Rua Napoleão de Barros 925, 04024-002 São Paulo SP (Fone 1155751677 a/c Valéria; e-mail valeria@psicobio.epm.br).

NEUROCIRURGIA: REUNIÕES DE DISCUSSÃO DE CASOS. O Departamento de Neurocirurgia da Associação Paulista de Medicina (APM) promove reuniões de discussão de casos, como parte das atividades de sua Diretoria. Esta é composta atualmente pelos colegas: Sérgio Listik, Presidente; J C Veiga, Diretor de Eventos; A C Montanaro, Secretário; J Adorno, Tesoureiro; A Andrade, Diretor Cien- tífico. As reuniões se realizam na sede da APM e para o restante deste ano estão programadas os seguintes temas, em: 21 de setembro, trauma raquimedular, tratamento neurocirúrgico das siringomielias. Informes e inscrições: Setor de Eventos Científicos da APM (www.apm.org.br): Avenida Brigadeiro Luís Antonio 278, 01318-901 São Paulo SP (Fone 113188 4252; e-mail patricia@apm.org.br).

III CONGRESSO LATINO-AMERICANO DE NEUROFISIOLOGIA CLÍNICA / IX JORNADA BRASILEIRA. De 11 a 14 setembro 2002 em Porto Seguro, Bahia (Arraial d'Ajuda) tem lugar este histórico evento em que, pela primeira vez, se reunem os congressos da Sociedade Brasileira de Neurofisiologia Clínica (SBENC) e o Capítulo Latino-Americano da Federação Internacional (IFCN). Temas: epilepsias, neuropatias periféricas, dor neuropática, esclerose lateral amiotrófica, miastenia gravis, potenciais evocados, estimulação magnética transcraniana, bloqueios de condução, eletromiografia quantitativa, esclerose múltipla, doenças do sono, neuropatias hereditárias, distúrbios de movimento, demências, monitorização cirúrgica, doenças priônicas, neuroplasticidade, magnetoencefalografia. Informes e inscrições na Secretaria Executiva (www.sbnc.org.br): Caixa Postal 2488, 30141-970 Belo Horizonte MG, Brasil (Fax 55313241 7893; e-mail 3clanfc@sbnc.org.br).

CONGRESSO PAULISTA DE NEUROCIÊNCIAS. De 19 a 21 setembro 2002 em Santos SP (SESC). Será uma oportunidade inegualável para que neurologistas, neurocirurgiões, psiquiatras, psicólogos, geriatras, fisioterapeutas, fonoaudiólogos e demais profissionais de diversas disciplinas interajam, troquem suas experiências sobre as ciências do sistema nervoso. O programa científico foi rigorosamente idealizado e terá participação de convidados estrangeiros e brasileiros, sob a coordenação do Dr. Edson José Amâncio. Temas Livres: inscrição até 29 julho 2002. Informes e inscrições: Golden Gate Eventos e Turismo, Rua Francisco Rocha 1657, 80730-390 Curitiba PR (fone 41 336 1288; fax 41335 6192; e-mail golgen@ggb.com.br; site oficial www.neurosp.com.br).

XI JORNADA DE PSIQUIATRIA DO ESTADO DO RIO DE JANEIRO. De 26 a 28 setembro 2002 na cidade do Rio de Janeiro (Academia Nacional de Medicina, Avenida General Justo 365). Esta jornada tem o patrocínio da Associação Psiquiátrica do Estado do Rio de Janeiro (APERJ) e é presidida pela colega Vera Lemgruber. Tema principal: "a psiquiatria no terceiro milênio: a integração cérebro-mente". Informes e inscrições: APERJ, Avenida Nossa Senhora Copacabana 613 / 1002, 22050-000 Rio de Janeiro RJ (Fax 212548 9439. E-mail aperj@centroin.com.br). 
XX CONGRESSO BRASILEIRO DE NEUROLOGIA. De 28 setembro a 3 outubro 2002, em Florianópolis (Centro Sul de Convenções). Este congresso é o evento máximo da Academia Brasileira de Neurologia (ABN) e sua Comissão Organizadora conta com o colega Paulo Norberto Discher de Sá como Presidente. Temas oficiais: distúrbios dos movimentos, epilepsia, doenças cerebrovasculares, cefaléias, neuropatias periféricas e miopatias, neurologia infantil, neuroinfectologia, neurogenética, doenças desmielinizantes, neurotoxicologia, neuroepidemiologia, sono, neuro UTI, neuroimunologia, neurooncologia, demências e distúrbios cognitivos. Temas livres: inscrição até 5 maio 2002. Informes e inscrições na ABN (www.abneuro. com.br) ou na Secretaria Executiva: Malu Losso, Rua Pinto Gonçalves 53, 05005-010 São Paulo SP (Fax 113864 4673; e-mailmlosso@uol.com.br).

AMERICAN NEUROLOGICAL ASSOCIATION (ANA): $127^{\text {th }}$ ANNUAL MEETING. De 13 a 16 outubro 2002 na cidade de New York (Marriott Marquis) realiza-se a reunião anual da ANA. Informes e inscrições: ANA, 5841 Cedar Lake Road Suite 204, Minneapolis MN 55416 USA. Fax 19525456073.

V JORNADAS NACIONALES DE NEUROLOIA TROPICAL Y NEUROINFECCION. De 24 a 26 outubro 2002 em Maracay, Venezuela (Hotel Pipo Ingernacional) realizamse estas já tradicionais jornadas. Elas são patrocinadas pela Sociedade Venezolana de Neurología (SVN) e têm o apoio da ASODIAM - Hospital Central de Maracay. Informes e inscrições: SVN (Fax 58212731 3093); ASODIAM (Fax 58243242 7943. E-mail asodiam@icinet.com.ve).

$6^{\text {th }}$ CONGRESS OF THE EUROPEAN FEDERATION OF NEUROLOGICAL SOCIETIES (EFNS). De 26 a 29 outubro 2002 em Viena (Wiener Medizinsche Akademie). Wofgang Grisold preside a Comissão de Organização. Temas:AVC, miopatias distais, infeções do SNC, desordens do movimento, esclerose múltipla, gliomas, epilepsia, demência, cefaléia. Informes e inscrições: Mrs Elisabeth Ribar,-Maurer, Alserstrasse 4, A-1090 Wien, Ausria. Fax 4314078274. E-mail: efns2002@medacad.org

$X$ CURSO EN ESPAÑOL DE LA ACADEMIA AMERICANA DE NEUROLOGÍA (AAN). De 24 a 27 novembro 2002 em San Juán Caribe Hilton. Este curso é patrocinado pela AAN com a SUNY Upstate Medical University (Syracuse NY, USA). O temário inclui: acidente vascular cerebral, demência, transtornos do sono, epilepsia, doença de Parkinson, dor. Informes e inscrições: SUNY Office of Continuing Education (www.upstate.edu/cme), a/c Nancy Haus - Program Coordinator, 250 Harrison Street Suite 303, Syracuse NY 13210 USA (Fax 1315464 4422).

IV CONGRESO VENEZOLANO DE EPILEPSIA, II CONGRESO DE LA ACADEMIA NEUROLÓGICA DE LOS PAÍSES BOLIVARIANOS, III JORNADAS DE NEUROPSIQUIATRÍA, XI JORNADAS NACIONALES DE EPILEPSIA, VII JORNADAS DE NEUROPEDIATRÍA. De 26 a 29 de março 2003 em Barquisimeto (Hotel Hilton) estes eventos neurológicos venezuelanos serão realizados conjuntamente, sob a égide da Universidad Centroccidental Lisandro Alvarado. Informes e inscrições com os colegas: Agustín D'Onghyia (email dongagus@telcel.net.ve) e Jorge Posadas E (e-mail jposadase@cantv.net).

ISI THOMSON NEWS RELEASE. June 7 anuncia que o ISI Web of Knowldedge expande seu conteúdo com o acréscimo da base de dados de Food Science and Technology Abstracts (FSTA ${ }^{\mathrm{TM}}$ ). June 7 noticia a parceria do ISI com WebFeat (Prism ${ }^{\mathrm{TM}}$ ) paa acesso de suas bases de dados utilizando uma só interface. June 17 informa sobre o acordo de ISI e Dartmouth College Library para estabelecer um portal integrado de pesquisa. Para detalhes, contatuar Rodney Yancey (rodney.yancey@isiresearchsoft. com) ou o ISI (www.isinet.com), 3501 Market Street, Philadelphia, PA 19104 USA (FAX 1215243 2235). 